\title{
Unraveling and Manipulating of NADH Oxidation by Photo-generated Holes
}

\author{
Shaohua Zhang, ${ }^{3,4}$ Jiafu Shi, ${ }^{1,2,6 *}$ Yixuan Chen, ${ }^{3}$ Qian Huo ${ }^{3,4}$, Weiran $\mathrm{Li}^{1}$, Yizhou Wu, ${ }^{3,4}$ Yiying Sun, ${ }^{3,4}$ \\ Yishan Zhang, ${ }^{3,4}$ Xiaodong Wang, ${ }^{5}$ Zhongyi Jiang ${ }^{2,3,4,6 *}$ \\ ${ }^{1}$ School of Environmental Science and Engineering, Tianjin University, Tianjin 300072, China \\ ${ }^{2}$ State Key Laboratory of Bioreactor Engineering, East China University of Science and Technology, Shanghai, China \\ ${ }^{3}$ Key Laboratory for Green Chemical Technology of Ministry of Education, School of Chemical Engineering and \\ Technology, Tianjin University, Tianjin 300072, China \\ ${ }^{4}$ Collaborative Innovation Center of Chemical Science and Engineering (Tianjin), Tianjin 300072, China \\ ${ }^{5}$ Department of Engineering, Lancaster University, Lancaster LA1 4YW, United Kingdom \\ ${ }^{6}$ Joint School of National University of Singapore and Tianjin University, International Campus of Tianjin University, Binhai \\ New City, Fuzhou, 350207, China
}

KEYWORDS: Photoenzymatic coupled catalysis, Photocatalytic NADH oxidation, Fragmentation, ADP-ribose, Dopamine

\begin{abstract}
Photoenzymatic coupled catalysis, integrating semiconductor photocatalysis and enzymatic catalysis, exhibits great potential for light-driven synthesis. To make photocatalyst and enzyme at play concertedly, nicotinamide-based cofactors have been widely used as electron carrier. However, these cofactors are easily oxidized into enzymatically inactive form by photo-generated holes. Herein, oxidation mechanism of $\mathrm{NADH}$, one typical nicotinamide-based cofactor, by photo-generated holes was reported. With $\mathrm{CdS}, \mathrm{g}-\mathrm{C}_{3} \mathrm{~N}_{4}$ and $\mathrm{BiVO}_{4}$ as hole generators, $\mathrm{NADH}$ is oxidized into $\mathrm{NAD}^{+}$or fragmented into ADP-ribose derivatives through multi-step electron transfer. Importantly, fragmentation reaction is inhibited with dopamine and neutral red to coordinate electron transfer between NADH and photo-generated holes.
\end{abstract}

Heterogeneous chemical catalysis, homogeneous chemical catalysis and enzymatic catalysis are three major catalytic processes used for manufacturing fuels, chemicals, and materials etc. Complex catalytic systems integrating the merits of the above catalytic processes play a vital role in exploring new reaction routes and achieving superior performances. ${ }^{1-5}$ Typically, photoenzymatic coupled catalytic systems (PECCSs), which integrate the light harvesting capability of semiconductor photocatalyst and high activity/selectivity of enzyme, have already been utilized for $\mathrm{CO}_{2}$ reduction, $\mathrm{H}_{2} / \mathrm{O}_{2}$ evolution and biomass conversion etc. ${ }^{6-11}$ Photoenzymatic reactions generally consist of three steps: 1) light absorption and charge separation by photocatalyst, 2) electron transfer between photocatalyst and enzyme, and 3) substrate conversion by enzyme. As the connecter between photocatalyst and enzyme, the electron transfer step determines the overall efficiency of photoenzymatic reaction. ${ }^{12-13}$ To harness the natural binding affinity and electron transfer mechanism of enzyme, nicotinamide-based cofactors, particularly $\mathrm{NADH} / \mathrm{NAD}^{+}$, have been widely used as the "electron shuttle" to coordinate the electron transfer between photocatalyst and enzyme. ${ }^{6,14-16}$ At present, the interaction between photo-generated electrons of photocatalyst and $\mathrm{NAD}^{+}$has been well elucidated, permitting the selective transduction of electronic energy into chemical energy of NADH for enzymatic conversion. ${ }^{17}$ However, the interaction between photo-generated holes, the antiparticle of electrons, and $\mathrm{NADH}$, which involves both interfacial electron transfer and solution radical chemistry, remains much less resolved. ${ }^{18-21}$ During the multiple interfacial electron transfer, NADH will not only be oxidized into the enzymatically active
$\mathrm{NAD}^{+}$, but also some unknown products from the active intermediate radicals. This would reduce the amount of effective electron shuttle $\left(\mathrm{NADH} / \mathrm{NAD}^{+}\right)$between photocatalyst and enzyme, imparting low turnover numbers (TNs) of PECCSs. ${ }^{12}$ Expounding the interaction between $\mathrm{NADH}$ and photo-generated holes, and manipulating NADH oxidation pathway could shed light on designing highly efficient PECCSs.

To evaluate the interaction between photo-generated holes and NADH, CdS particles (diameter: 232.5 $\pm 12.8 \mathrm{~nm}$ ) was used as a model photocatalyst (Figure S1). The experiments were conducted in anaerobic phosphate buffer to exclude the influence of oxygen derived radicals on NADH. In the absence of $\mathrm{CdS}, 96.1 \pm 0.1 \% \mathrm{NADH}$ was retained after $60 \mathrm{~min}$ illumination $(\geq 420 \mathrm{~nm}$ ). When $\mathrm{CdS}$ was added, NADH content got dramatically decreased to $30.5 \pm 5.7 \%$ (Figure 1a) and simultaneously $0.58 \pm 0.07 \mu \mathrm{mol} \mathrm{H}_{2}$ was produced (Figure S2). The mole ratio between $\mathrm{H}_{2}$ and oxidized $\mathrm{NADH}$ was $0.84: 1.00$, suggesting that $\mathrm{NADH}$ oxidation was accompanied by $\mathrm{H}_{2}$ evolution (Figure 1b). Moreover, the normalized apparent quantum yield of NADH oxidation coincided with the light absorption of $\mathrm{CdS}$ (Figure S3), confirming the photocatalytic NADH oxidation. Since the valence band (VB) potential of $\mathrm{CdS}$ was $0.86 \mathrm{~V}$ vs saturated calomel electrode (SCE) which could not oxidize water to $\bullet \mathrm{OH}$ in neutral phosphate buffer, NADH should be directly oxidized by 


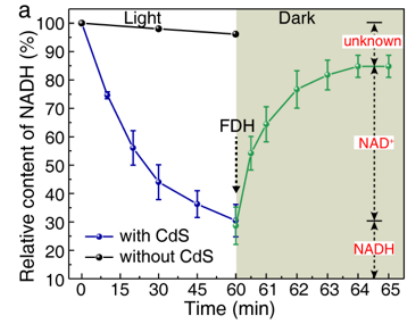

b
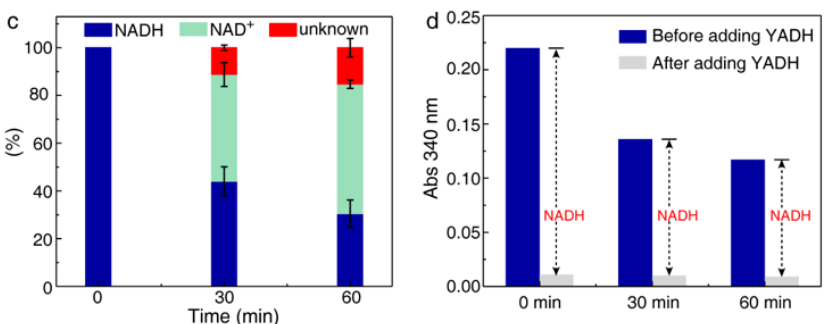

Figure 1. (a) NADH oxidation by $\mathrm{CdS}$ and $\mathrm{NAD}^{+}$reduction by FDH. (b) Schematic photocatalytic NADH oxidation and enzymatic product analysis. (c) Percentage of different species in NADH oxidation product. (d) NADH oxidation by YADH. Reaction condition for (a), Light stage: $[\mathrm{CdS}]=1 \mathrm{mg} \mathrm{mL}^{-1}$, $[\mathrm{NADH}]=1 \mathrm{mM}$, [phosphate buffer $(\mathrm{pH} 7.0)]=50 \mathrm{mM}, \mathrm{T}=$ $30{ }^{\circ} \mathrm{C}$, Xe-lamp $(\lambda \geq 420 \mathrm{~nm})$, anaerobic condition. Dark stage: $[\mathrm{FDH}]=0.02 \mathrm{mg} \mathrm{mL}^{-1}$, [Formate] $=10 \mathrm{mM}$, [phosphate buffer $(\mathrm{pH} 7.0)]=50 \mathrm{mM}$ with 100 times diluted reaction solution. Reaction condition for (d), $[\mathrm{YADH}]=1 \mu \mathrm{g} \mathrm{mL}^{-1}$, $[$ formaldehyde $]=10 \mathrm{mM}$, [phosphate buffer $(\mathrm{pH} 7.0)]=50$ $\mathrm{mM}$ with 30 times diluted reaction solution. Error bars indicated standard deviation of duplicate experiments $(n=2)$.

photo-generated holes of CdS. ${ }^{22-23} \mathrm{CdS}$ kept stable during the reaction, where only $1.04 \mathrm{ug} \mathrm{mL} \mathrm{mL}^{-1} \mathrm{Cd}$ was detected in the reaction solution by ICP-MS. To elucidate the reaction mechanism, NADH oxidation product was analyzed by formate dehydrogenase (FDH), which could fully reduce $\mathrm{NAD}^{+}$to NADH by consuming formate (Figure 1b). ${ }^{24}$ As
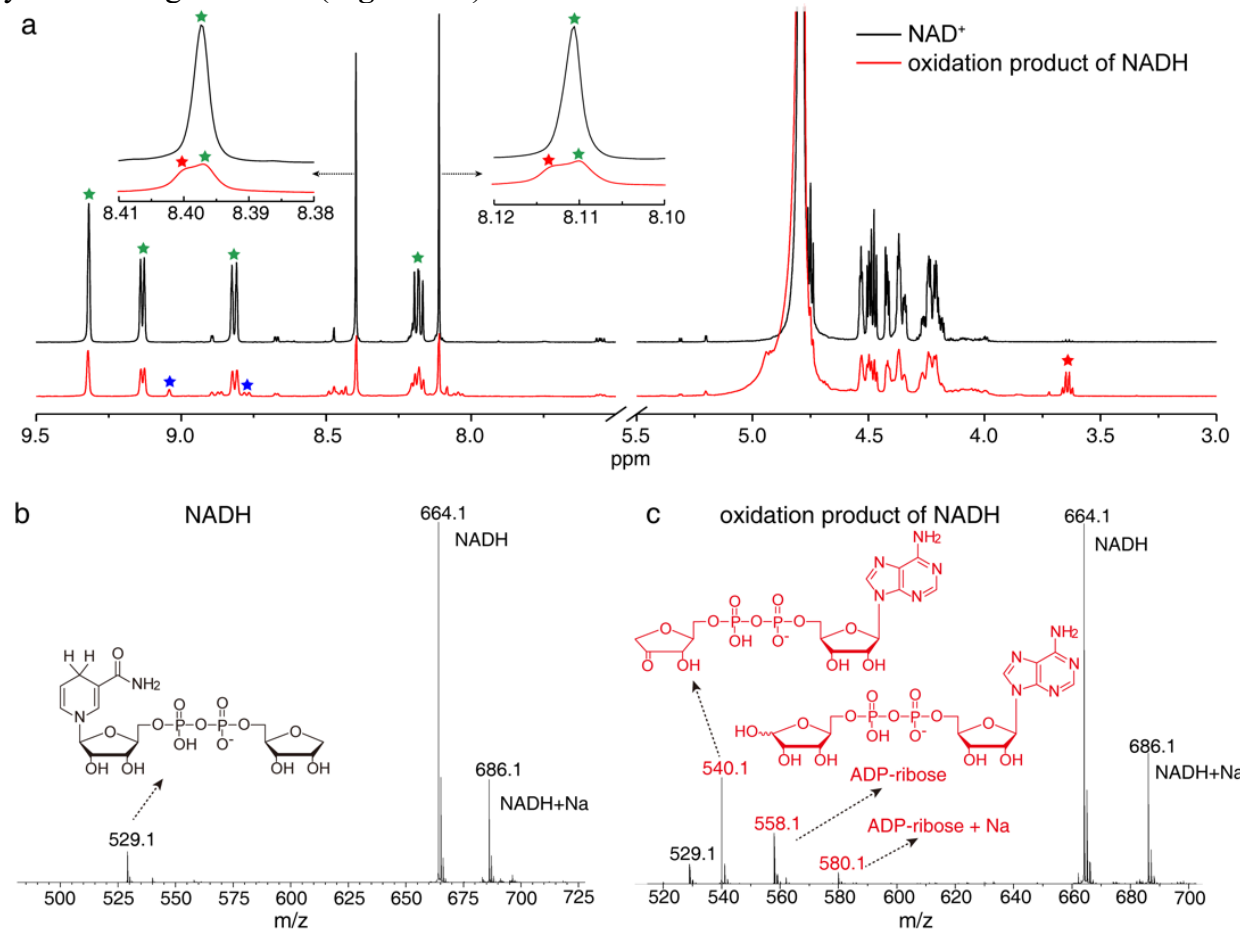

Figure 2. (a) ${ }^{1} \mathrm{H}$ NMR of $\mathrm{NAD}^{+}(25 \mathrm{mM})$ and photocatalytic oxidation product of NADH $(25 \mathrm{mM})$ in $\mathrm{D}_{2} \mathrm{O}$ with phosphate buffer $(50 \mathrm{mM})$ at $\mathrm{pD}=7.0$. $\star, \star$ and $\star$ represented signals of adenosine diphosphate ribose (ADP-ribose), nicotinamide, and NAD ${ }^{+}$, respectively. The negative ion ESI-MS of (b) NADH and (c) photocatalytic oxidation product of NADH. shown in Figure 1a, after the addition of FDH, NADH content can only reach $84.8 \pm 3.9 \%$. The decrease of initially added NADH suggested that unknown product other than NAD ${ }^{+}$was formed during photocatalytic NADH oxidation. Moreover, the content of unknown product got increased with the consumption of NADH, evidencing its formation during NADH oxidation (Figure 1c). Interestingly, the formation of unknown product was not specific for CdS, which was also observed for two other widely used semiconductor photocatalysts, g- $\mathrm{C}_{3} \mathrm{~N}_{4}$ and $\mathrm{BiVO}_{4}$ (Figure S4 and S5). When incubating $\mathrm{NAD}^{+}$with $\mathrm{CdS}$, equal amount of $\mathrm{NADH}$ was generated by FDH at different illumination time (Figure S6). This demonstrated that $\mathrm{NAD}^{+}$was not degraded by $\mathrm{CdS}$, and the unknown product was generated during NADH oxidation.

The formation of unknown product suggested that $\mathrm{NADH}$ oxidation by photo-generated holes did not follow the one-step hydride transfer where only $\mathrm{NAD}^{+}$would be generated, but the three-step electron-proton-electron transfer. ${ }^{25}$ For three-step oxidation, NAD radicals (NAD•) would be formed, which may undergo dimerization or fragmentation to generate the unknown products. The dimers of NAD • ((NAD) $\left.)_{2}\right)$ exhibited absorption band at $340 \mathrm{~nm}$ with a extinction coefficient similar to NADH $\left(6550\right.$ vs 6220). ${ }^{26}$ If (NAD) 2 was the unknown product, the absorption at $340 \mathrm{~nm}$ should belong to both the residual NADH and newly formed (NAD) $)_{2}$. To determine if (NAD) 2 was the unknown product, the oxidation solution was analyzed by yeast alcohol dehydrogenase (YADH), which could irreversibly oxidize $\mathrm{NADH}$ into $\mathrm{NAD}^{+}$during the conversion of formaldehyde into methanol, while not $(\mathrm{NAD})_{2}{ }^{27}$ As shown in Figure 1d, after adding YADH into the mixture of formaldehyde and NADH oxidation solution, the absorption band at $340 \mathrm{~nm}$ decreased to a similar level. This indicated that (NAD) $)_{2}$ did not contribute to the unknown product. As was reported, (NAD) $)_{2}$ was not stable under visible light illumination, which would undergo disproportionation to 


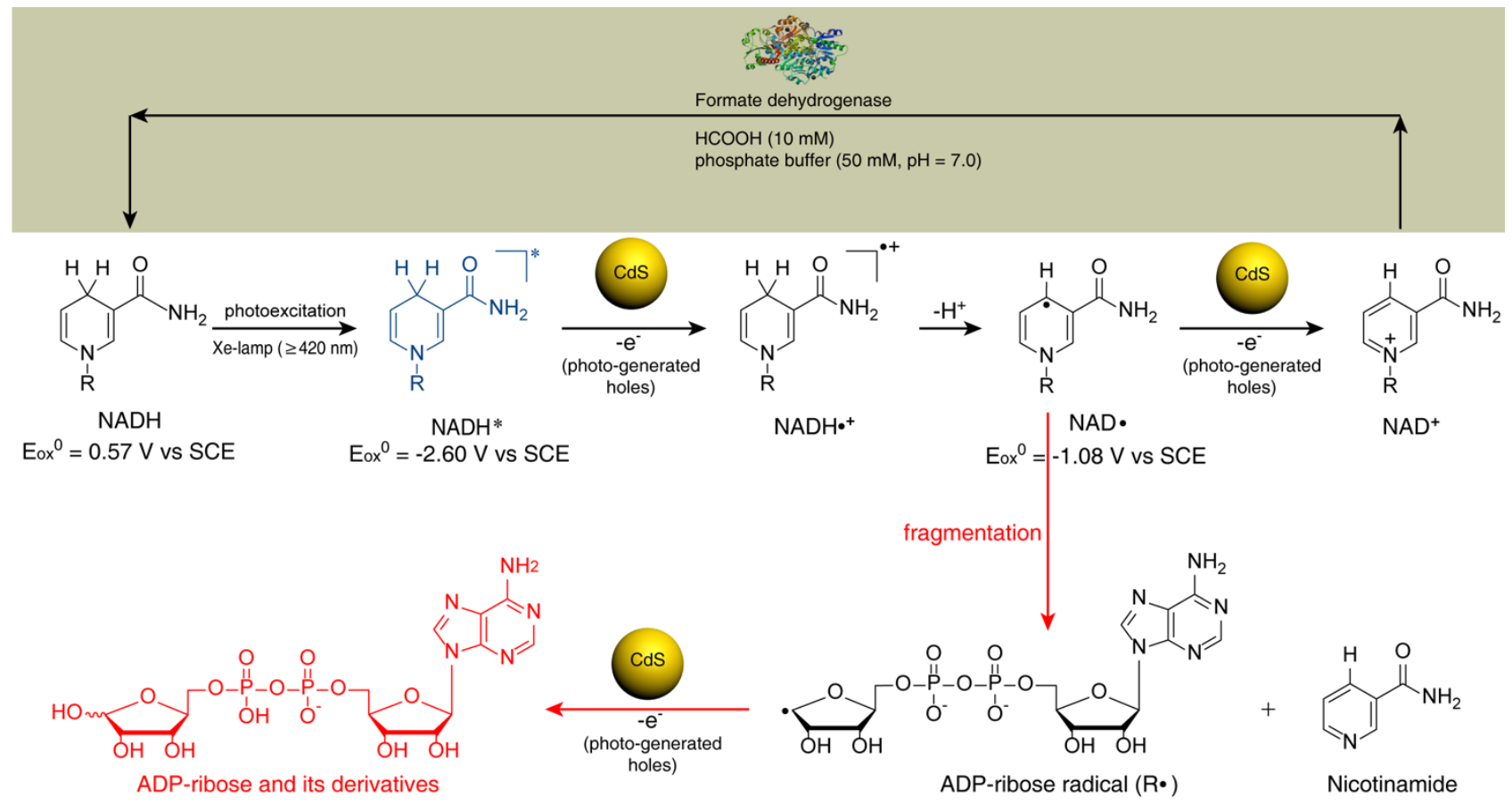

Figure 3. NADH oxidation mechanism by photo-generated holes on CdS particles.

$\mathrm{NADH}$ and $\mathrm{NAD}^{+}$, or oxidation to $\mathrm{NAD}^{+}$by photo-generated holes. $^{28-29}$ Thus, (NAD) 2 did not accumulate during NADH oxidation. Besides dimerization, NAD - would also undergo fragmentation. ${ }^{30}$ To verify the reaction mechanism, NADH oxidation products were analyzed by ${ }^{1} \mathrm{H}$ NMR and negative ion Electrospray Ionization Mass Spectrometry (ESI-MS). As shown in Figure 2a, besides the typical peaks of NAD ${ }^{+}$ (Figure S7), some new peaks, marked by red and blue star, were observed (Figure S8), which was consistent with the characteristic ${ }^{1} \mathrm{H}$ NMR peaks of ADP-ribose and nicotinamide..$^{31-32}$ This suggested that the unknown product might be ADP-ribose and nicotinamide arisen from the fragmentation of NAD $\bullet$. From the ESI-MS spectra, peaks at $\mathrm{m} / \mathrm{z}$ 540.1, 558.1 and 580.1 corresponding to ADP-ribose and its derivatives were detected (Figure $2 \mathrm{~b}$ and $\mathrm{c}$ ). This further evidenced the fragmentation of $\mathrm{NADH}$ into ADP-ribose and its derivatives during photocatalytic NADH oxidation. The observation of NADH peak in Figure 2c was probably arisen from the reduction of $\mathrm{NAD}^{+}$during ESI-MS measurement. ${ }^{33}$ Besides $\mathrm{CdS}$, the unknown products generated by $\mathrm{g}-\mathrm{C}_{3} \mathrm{~N}_{4}$ and $\mathrm{BiVO}_{4}$ were also determined as ADP-ribose and its derivatives (Figure S9 and S10). This manifested that the property of semiconductor photocatalyst was not the major factor that determined the NADH oxidation pathway. As reported previously, NADH can be excited into strong single-electron reductant by visible light through two-photon absorption (Figure S11). ${ }^{21}$ This will induce the single electron oxidation of NADH into NAD•, which was further fragmented into ADP-ribose and its derivatives (unknown product).

Based on the above discussion, we proposed the NADH oxidation mechanism by photo-generated holes (Figure 3). Under visible light illumination, both NADH and CdS were excited. The excited state of NADH $\left(\mathrm{NADH}^{*}\right)$ was a strong single-electron reductant with oxidation potential of $-2.60 \mathrm{~V}$ vs SCE, which went through single electron oxidation by photo-generated holes. ${ }^{21}$ The generated radical cations $\mathrm{NADH}^{\bullet}{ }^{+}$then got deprotonated into NAD• in phosphate buffer ( $\mathrm{pH} 7.0) \cdot{ }^{34,35} \mathrm{NAD} \cdot$ with an oxidation potential of $-1.08 \mathrm{~V}$ vs SCE was oxidized to enzymatically active $\mathrm{NAD}^{+}$ by the photo-generated holes or fragmented into ADP-ribose radical and nicotinamide. ${ }^{30,36}$ ADP-ribose radical was then converted to ADP-ribose and its derivatives by photo-generated holes. The fragmentation of NAD• contributed to the formation of unknown product. This special oxidation pathway should rely on the simultaneous photoexcitation of NADH and CdS. To selectively excite $\mathrm{NADH}$ and $\mathrm{CdS}$, two beams of $340 \mathrm{~nm}$ and $\geq 420 \mathrm{~nm}$ were used for NADH oxidation. The two beams experiment further confirmed the role of dual excitation in NADH oxidation (Figure S12). More detailed discussion could be found in the Supporting Information.
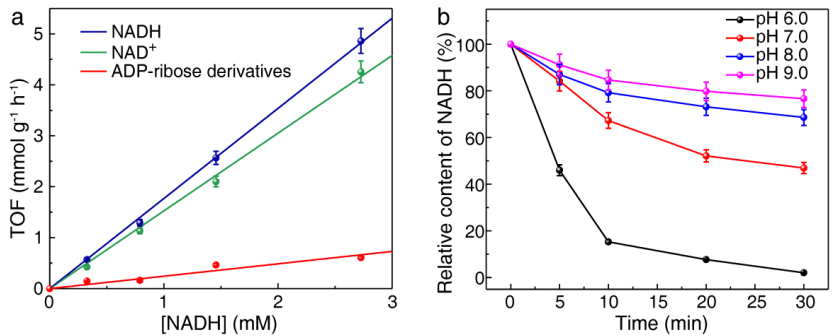

Figure 4. Kinetics of photocatalytic NADH oxidation. (a) Plots of TOF for the oxidation of NADH, formation of $\mathrm{NAD}^{+}$and ADP-ribose derivatives vs the concentration of $\mathrm{NADH}$. (b) Oxidation curves vs $\mathrm{pH}$ values of the solution.

To better understand photocatalytic NADH oxidation, the reaction kinetics were examined. As shown in Figure 4a, the turnover frequency (TOF) of NADH increased linearly with $\mathrm{NADH}$ concentration, suggesting the first-order kinetics of photocatalytic NADH oxidation. This should be arisen from the full excitation of NADH under $100 \mathrm{~mW} \mathrm{~cm}^{-2}$ visible light (Figure S11). Moreover, TOF for the generation of $\mathrm{NAD}^{+}$ and ADP-ribose derivatives also exhibited linear dependence on NADH concentration. The observed rate constant $\left(\mathrm{k}_{\mathrm{obs}}\right)$ of NADH oxidation was calculated to be $4.9 \times 10^{-4} \mathrm{~s}^{-1}$ at $\mathrm{pH} 7.0$ and $303 \mathrm{~K}$. This was much lower than the previously reported deprotonation rate constant of $\mathrm{NADH}^{\bullet}{ }^{+}\left(3.5 \times 10^{6}\right.$ $\mathrm{s}^{-1}$ ), suggesting that deprotonation was not the rate-limiting step. ${ }^{34,35}$ Considering the high reactivity of NAD•, we speculated that oxidation of $\mathrm{NADH}$ to $\mathrm{NADH} \bullet^{+}$was the 
rate-limiting step. ${ }^{36}$ To verify this hypothesis, the photocatalytic NADH oxidation was performed in phosphate buffer with different $\mathrm{pH}$ values $(6.0-9.0)$. As shown in Figure $4 b$, the NADH oxidation rate got gradually decreased with the increase of $\mathrm{pH}$. Since alkaline condition was beneficial for $\mathrm{NADH}^{\bullet}+$ deprotonation, the decreased oxidation rate under high $\mathrm{pH}$ values further confirmed that deprotonation step was not the rate-limiting step. In general, NADH oxidation by photo-generated holes mainly included the following three steps: 1) adsorption of NADH onto CdS, 2) electron injection from NADH to photo-generated holes of $\mathrm{CdS}, 3)$ and desorption of oxidation product from CdS. The change of $\mathrm{pH}$ should primarily alter NADH adsorption. Due to the low isoelectric point of CdS (3.03), the negative potential of CdS kept stable at around $-17 \mathrm{mV}$ between $\mathrm{pH}$ 6.0-9.0. ${ }^{37}$ However, for NADH, the phosphate group got a $\mathrm{pKa}$ value of $6.82 .{ }^{38}$ When $\mathrm{pH}$ was increased from 6.0 to 7.0 , phosphate group would deprotonate, resulting in negatively charged NADH. Electrostatic repulsion between NADH and $\mathrm{CdS}$ would inhibit the adsorption of $\mathrm{NADH}$, and reduce $\mathrm{NADH}$ oxidation rate. When $\mathrm{pH}$ value was further increased from 7.0 to 9.0 , the oxidation rate only decreased slightly (Figure S13). This was consistent with the unchanged potential of NADH between $\mathrm{pH}$ 7.0-9.0, which confirmed that the adsorption of NADH during the conversion of $\mathrm{NADH}$ into $\mathrm{NADH}^{{ }^{+}}$was the rate-limiting step in photocatalytic NADH oxidation. ${ }^{39}$

During photocatalytic NADH oxidation, both enzymatically active $\mathrm{NAD}^{+}$and inactive ADP-ribose derivatives were generated. This would reduce the effective electron shuttle (NADH/NAD ${ }^{+}$) between photocatalyst and enzyme. To inhibit the generation of ADP-ribose derivatives, one step hydride transfer instead of three step electron-proton-electron transfer should be constructed between NADH and photo-generated holes. Firstly, dopamine, a commonly used catalyst for electrochemical $\mathrm{NADH}$ oxidation, was adopted to mediate $\mathrm{NADH}$ oxidation. ${ }^{40}$ The interconversion between dopamine and dopamine quinone should allow the one-step hydride transfer between NADH and photo-generated holes. ${ }^{41}$ As shown in Figure 5a, after adding $2 \mathrm{mg} \mathrm{mL}^{-1}$ dopamine, the percentage of ADP-ribose derivatives in the oxidation solution decreased from $41.2 \pm 3.9 \%$ to $10.2 \pm 2.4 \%$. This confirmed the role of dopamine in mediating one-step hydride transfer between NADH and photo-generated holes. Moreover, $\mathrm{NADH}$ oxidation rate got increased with dopamine concentration (Figure 5b). This should be arisen from the better interfacial affinity of dopamine bearing catechol groups than $\mathrm{NADH}$, which accelerated the interfacial electron transfer and subsequent NADH oxidation (Figure 5c). Moreover, dopamine kept stable during photocatalytic $\mathrm{NADH}$ oxidation, which did not polymerize into polydopamine as verified by UV-vis spectrum (Figure S14). However, dopamine was usually not stable under aerobic condition, which restricted its use for aerobic enzyme. Inspired by flavin adenine dinucleotide (FAD) mediated NADPH oxidation, neutral red, a stable chemical under both anaerobic and aerobic condition, was used to mediate NADH oxidation (Figure S15). ${ }^{42}$ As shown in Figure 5a, after adding $2 \mathrm{mg} \mathrm{mL}^{-1}$ neutral red, only $1.8 \pm 0.1 \%$ of the initially added NADH was fragmented into ADP-ribose derivatives. Neutral red and dopamine successfully mediated NADH oxidation to $\mathrm{NAD}^{+}$, other than ADP-ribose derivatives.
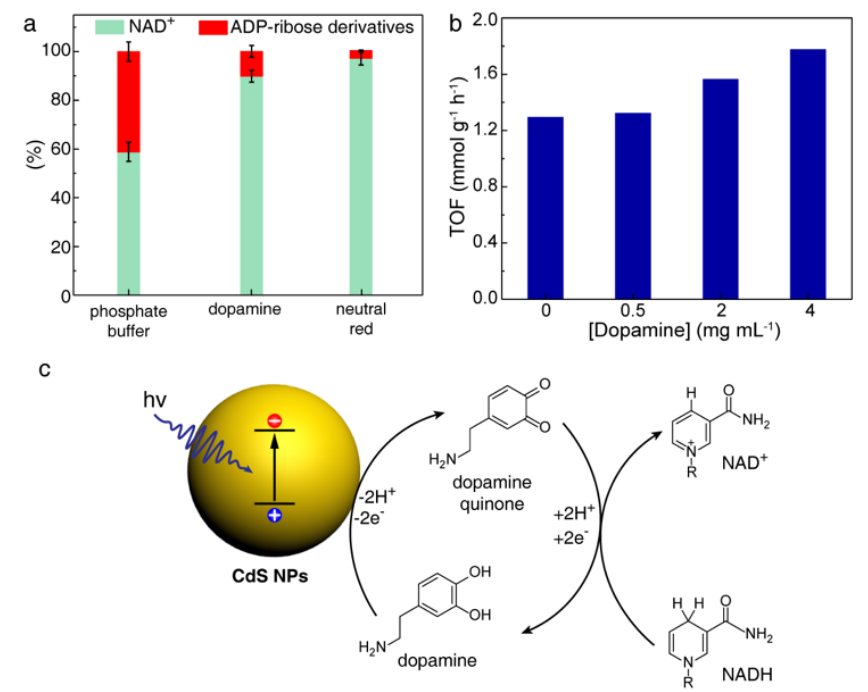

Figure 5. Photocatalytic $\mathrm{NADH}$ oxidation by $\mathrm{CdS}$ and dopamine. (a) Relative percentage of $\mathrm{NAD}^{+}$and ADP-ribose derivatives after $100 \% \mathrm{NADH}$ oxidation by CdS with $2 \mathrm{mg}$ $\mathrm{mL}^{-1}$ dopamine or $2 \mathrm{mg} \mathrm{mL}^{-1}$ neutral red. (b) Plot of NADH oxidation TOF vs dopamine concentration. (c) Dopamine coordinated electron transfer between NADH and CdS.

In summary, NADH oxidation mechanism by photo-generated holes were proposed and verified in this study. NADH was converted to $\mathrm{NAD}^{+}$or fragmented into ADP-ribose derivatives by photo-generated holes through multistep electron transfer. The conversion of NADH into $\mathrm{NADH}^{\bullet}{ }^{+}$was the rate-determining step of photocatalytic $\mathrm{NADH}$ oxidation. The fragmentation reaction led to the decrease of the total amount of enzymatically active NADH and $\mathrm{NAD}^{+}$. By using dopamine and neutral red as the mediator, the percentage of fragmentation products significantly decreased from $41.2 \pm 3.9 \%$ to $10.2 \pm 2.4 \%$ and $1.8 \pm 0.1 \%$. Hopefully, this study could afford some in-depth understandings for the design of PECCSs involving nicotinamide-based cofactors.

\section{AUTHOR INFORMATION}

Corresponding Authors

*E-mail: shijiafu@tju.edu.cn (JS); zhyjiang@tju.edu.cn (ZJ)

Author Contributions

The manuscript was written through contributions of all authors. I All authors have given approval to the final version of the manuscript.

\section{Notes}

The authors declare no competing financial interests.

\section{ASSOCIATED CONTENT}

Supporting Information. Experimental Methods; SEM image of CdS particles; Photocatalytic $\mathrm{H}_{2}$ evolution; Action spectrum; Photocatalytic NADH oxidation by $\mathrm{g}-\mathrm{C}_{3} \mathrm{~N}_{4}$ and $\mathrm{BiVO}_{4}$; photocatalytic oxidation product of $\mathrm{NAD}^{+} ;{ }^{1} \mathrm{H}$ NMR of $\mathrm{NAD}^{+}$and photocatalytic oxidation products of NADH; ${ }^{1} \mathrm{H}$ NMR and ESI-MS of photocatalytic NADH oxidation products by $\mathrm{g}_{-} \mathrm{C}_{3} \mathrm{~N}_{4}$ and $\mathrm{BiVO}_{4}$; Photoluminescence of NADH; Photocatalytic NADH oxidation by two beams of light; Relative reaction rate of photocatalytic NADH oxidation in phosphate buffer with different $\mathrm{pH}$ values; UV-vis of dopamine; and NADPH oxidation by 
Ferredoxin-NADP ${ }^{+}$reductase. This material is available free of charge via the Internet at http://pubs.acs.org.

\section{ACKNOWLEDGMENT}

The authors thank the National Natural Science Funds of China $(21776213,21621004)$, Natural Science Fund of Tianjin (19JCYBJC19700), Open Funding Project of the National Key Laboratory of Biochemical Engineering (2015KF-03) and Open Funding Project of the State Key Laboratory of Bioreactor Engineering for financial support.

\section{REFERENCES}

1. Litman, Z. C.; Wang, Y.; Zhao, H.; Hartwig, J. F. Cooperative Asymmetric Reactions Combining Photocatalysis and Enzymatic Catalysis. Nature 2018, 560, 355-359.

2. Rudroff, F.; Mihovilovic, M. D.; Gröger, H.; Snajdrova, R.; Iding, H.; Bornscheuer, U. T. Opportunities and Challenges for Combining Chemo-and Biocatalysis. Nat. Catal. 2018, 1, 12-22.

3. Denard, C. A.; Bartlett, M. J.; Wang, Y.; Lu, L.; Hartwig, J. F.; Zhao, H. Development of a One-Pot Tandem Reaction Combining Ruthenium-Catalyzed Alkene Metathesis and Enantioselective Enzymatic Oxidation to Produce Aryl Epoxides. ACS Catal. 2015, 5, 3817-3822.

4. Kornienko, N.; Zhang, J. Z.; Sakimoto, K. K.; Yang, P.; Reisner, E. Interfacing Nature's Catalytic Machinery with Synthetic Materials for Semi-Artificial Photosynthesis. Nat. Nanotechnol. 2018, 13, 890-899.

5. Zhang, W.; Fernández-Fueyo, E.; Ni, Y.; van Schie, M.; Gacs, J.; Renirie, R.; Wever, R.; Mutti, F. G.; Rother, D.; Alcalde, M. Selective Aerobic Oxidation Reactions Using a Combination of Photocatalytic Water Oxidation and Enzymatic Oxyfunctionalizations. Nat. Catal. 2018, 1, 55-62.

6. Kuk, S. K.; Singh, R. K.; Nam, D. H.; Singh, R.; Lee, J. K.; Park, C. B. Photoelectrochemical Reduction of Carbon Dioxide to Methanol through a Highly Efficient Enzyme Cascade. Angew. Chem. Int. Ed. 2017, 56, 3827-3832.

7. Kuk, S. K.; Ham, Y.; Gopinath, K.; Boonmongkolras, P.; Lee, Y.; Lee, Y. W.; Kondaveeti, S.; Ahn, C.; Shin, B.; Lee, J. K. Continuous 3D Titanium Nitride Nanoshell Structure for Solar-Driven Unbiased Biocatalytic $\mathrm{CO}_{2}$ Reduction. $A d v$. Energy Mater. 2019, 9, 1900029.

8. Zhang, L.; Can, M.; Ragsdale, S. W.; Armstrong, F. A. Fast and Selective Photoreduction of $\mathrm{CO}_{2}$ to $\mathrm{CO}$ Catalyzed by a Complex of Carbon Monoxide Dehydrogenase, $\mathrm{TiO}_{2}$, and Ag nanoclusters. ACS Catal. 2018, 8, 2789-2795.

9. Hutton, G. A.; Reuillard, B.; Martindale, B. C.; Caputo, C. A.; Lockwood, C. W.; Butt, J. N.; Reisner, E. Carbon Dots as Versatile Photosensitizers for Solar-Driven Catalysis with Redox Enzymes. J. Am. Chem. Soc. 2016, 138, 16722-16730.

10. Bachmeier, A.; Murphy, B. J.; Armstrong, F. A. A Multi-Heme Flavoenzyme as a Solar Conversion Catalyst. $J$. Am. Chem. Soc. 2014, 136, 12876-12879.

11. Kim, J.; Lee, S. H.; Tieves, F.; Choi, D. S.; Hollmann, F.; Paul, C. E.; Park, C. B. Biocatalytic $\mathrm{C}=\mathrm{C}$ Bond Reduction through Carbon Nanodot-Sensitized Regeneration of NADH Analogues. Angew. Chem. Int. Ed. 2018, 57, 13825-13828.

12. Lee, S. H.; Choi, D. S.; Kuk, S. K.; Park, C. B. Photobiocatalysis: Activating Redox Enzymes by Direct or Indirect Transfer of Photoinduced Electrons. Angew. Chem., Int. Ed. 2018, 57, 7958-7985.
13. Wombwell, C.; Caputo, C. A.; Reisner, E. [NiFeSe]-Hydrogenase Chemistry. Acc. Chem. Res. 2015, 48, 2858-2865.

14. Ji, X.; Wang, J.; Kang, Y.; Mei, L.; Su, Z.; Wang, S.; Ma, G.; Shi, J.; Zhang, S. Enhanced Solar Energy Harvest and Electron Transfer through Intra-and Intermolecular Dual Channels in Chlorosome-Mimicking Supramolecular Self-Assemblies. ACS Catal. 2018, 8, 10732-10745.

15. Megarity, C. F.; Siritanaratkul, B.; Heath, R. S.; Wan, L.; Morello, G.; FitzPatrick, S. R.; Booth, R. L.; Sills, A. J.; Robertson, A. W.; Warner, J. H. Electrocatalytic Volleyball: Rapid Nanoconfined Nicotinamide Cycling for Organic Synthesis in Electrode Pores. Angew. Chem. Int. Ed. 2019, 58, 4948-4952.

16. Chen, Y.; Li, P.; Noh, H.; Kung, C. W.; Buru, C. T.; Wang, X.; Zhang, X.; Farha, O. K. Stabilization of Formate Dehydrogenase in a Metal-Organic Framework for Bioelectrocatalytic Reduction of $\mathrm{CO}_{2}$. Angew. Chem. Int. Ed. 2019, 58, 7682-7686.

17. Maenaka, Y.; Suenobu, T.; Fukuzumi, S. Efficient Catalytic Interconversion between $\mathrm{NADH}$ and $\mathrm{NAD}^{+}$ Accompanied by Generation and Consumption of Hydrogen with a Water-Soluble Iridium Complex at Ambient Pressure and Temperature. J. Am. Chem. Soc. 2012, 134, 367-374.

18. Mohanty, B.; Naik, K. K.; Sahoo, S.; Jena, B.; Chakraborty, B.; Rout, C. S.; Jena, B. K. Efficient Photoelectrocatalytic Activity of $\mathrm{CuWO}_{4}$ Nanoplates towards the Oxidation of NADH Driven in Visible Light. ChemistrySelect 2018, 3, 9008-9012.

19. Ryu, G. M.; Lee, M.; Choi, D. S.; Park, C. B. A Hematite-based Photoelectrochemical Platform for Visible Light-Induced Biosensing. J. Mater. Chem. B 2015, 3, 4483-4486.

20. Samantara, A. K.; Sahu, S. C.; Bag, B.; Jena, B.; Jena, B. $\mathrm{K}$. Photoelectrocatalytic Oxidation of NADH by Visible Light Driven Plasmonic Nanocomposites. J. Mater. Chem. A 2014, 2, 12677-12680.

21. Emmanuel, M. A.; Greenberg, N. R.; Oblinsky, D. G.; Hyster, T. K. Accessing Non-Natural Reactivity by Irradiating Nicotinamide-Dependent Enzymes with Light. Nature 2016, 540, 414-417.

22. Simon, T.; Bouchonville, N.; Berr, M. J.; Vaneski, A.; Adrovic, A.; Volbers, D.; Wyrwich, R.; Doblinger, M.; Susha, A. S.; Rogach, A. L.; Jackel, F.; Stolarczyk, J. K.; Feldmann, J. Redox Shuttle Mechanism Enhances Photocatalytic $\mathrm{H}_{2}$ Generation on Ni-Decorated $\mathrm{Cds}$ Nanorods. Nat. Mater. 2014, 13, 1013-1018.

23. Ran, J. R.; Gao, G. P.; Li, F. T.; Ma, T. Y.; Du, A. J.; Qiao, S. Z. $\mathrm{Ti}_{3} \mathrm{C}_{2}$ MXene Co-Catalyst on Metal Sulfide Photo-Absorbers for Enhanced Visible-Light Photocatalytic Hydrogen Production. Nat. Commun. 2017, 8, 10.

24. Willot, S. J. P.; Fernandez-Fueyo, E.; Tieves, F.; Pesic, M.; Alcalde, M.; Arends, I. W.; Park, C. B.; Hollmann, F. Expanding the Spectrum of Light-Driven Peroxygenase Reactions. ACS Catal. 2018, 9, 890-894.

25. Gebicki, J.; Marcinek, A.; Zielonka, J. Transient Species in the Stepwise Interconversion of $\mathrm{NADH}$ and $\mathrm{NAD}^{+}$. Acc. Chem. Res. 2004, 37, 379-386.

26. Burnett, R. W.; Underwood, A. L. A Dimer of Diphosphopyridine Nucleotide. Biochemistry 1968, 7, 3328-3333.

27. Ma, K.; Yehezkeli, O.; Park, E.; Cha, J. N. Enzyme Mediated Increase in Methanol Production from Photoelectrochemical Cells and $\mathrm{CO}_{2}$. ACS Catal. 2016, 6, 6982-6986.

28. Czochralska, B.; Szweykowska, M.; Shugar, D. Photochemical Redox Transformations of Dimers of NAD ${ }^{+}$ 
and N'-methylnicotinamide. Arch. Biochem. Biophys. 1980, 199, 497-505.

29. Bresnahan, W. T.; Elving, P. J. Spectrophotometric Investigation of Products Formed Following the Initial One-Electron Electrochemical Reduction of Nicotinamide Adenine Dinucleotide $\left(\mathrm{NAD}^{+}\right)$. Biochim. Biophys. Acta 1981, 678, 151-156.

30. Vitinius, U.; Schaffner, K.; Demuth, M.; Heibel, M.; Selbach, H. New Photoproducts from Irradiation of NADH with Near-UV Light. Chem. Biodivers. 2004, 1, 1487-1497.

31. Pennacchio, A.; Giordano, A.; Esposito, L.; Langella, E.; Rossi, M.; Raia, C. A. Insight into the Stereospecificity of Short-Chain Thermus Thermophilus Alcohol Dehydrogenase Showing Pro-S Hydride Transfer and Prelog Enantioselectivity. Protein Pept. Lett. 2010, 17, 437-443.

32. Jackson, M. D.; Denu, J. M. Structural Identification of 2'- and 3'-O-Acetyl-ADP-Ribose as Novel Metabolites Derived from the Sir2 Family of $\beta-\mathrm{NAD}^{+}$-Dependent Histone/Protein Deacetylases. J. Biol. Chem. 2002, 277, 18535-18544.

33. Khan, S. R.; Morgan, A. G.; Michail, K.; Srivastava, N.; Whittal, R. M.; Aljuhani, N.; Siraki, A. G. Metabolism of Isoniazid by Neutrophil Myeloperoxidase Leads to Isoniazid-NAD ${ }^{+}$Adduct Formation: a Comparison of the Reactivity of Isoniazid with Its Known Human Metabolites. Biochem. Pharmacol. 2016, 106, 46-55.

34. Zielonka, J.; Marcinek, A.; Adamus, J.; Gebicki, J. Direct Observation of NADH Radical Cation Generated in Reactions with One-Electron Oxidants. J. Phys. Chem. A 2003, 107, 9860-9864.

35. Kotani, H.; Ono, T.; Ohkubo, K.; Fukuzumi, S. Efficient Photocatalytic Hydrogen Evolution without an Electron Mediator Using a Simple Electron Donor-Acceptor Dyad. Phys. Chem. Chem. Phys. 2007, 9, 1487-1492.

36. Fukuzumi, S.; Inada, O.; Suenobu, T. Mechanisms of Electron-Transfer Oxidation of NADH Analogues and Chemiluminescence. Detection of the Keto and Enol Radical Cations. J. Am. Chem. Soc. 2003, 125, 4808-4816.

37. Zhang, L.; Niu, C. G.; Wen, X. J.; Guo, H.; Zhao, X. F.; Huang, D. W.; Zeng, G. M. A Facile Strategy to Fabricate Hollow Cadmium Sulfide Nanospheres with Nanoparticles-Textured Surface for Hexavalent Chromium Reduction and Bacterial Inactivation. J. Colloid Interface Sci. 2018, 514, 396-406.

38. Hill, C.; Harris, R. C.; Kim, H.; Harris, B.; Sale, C.; Boobis, L.; Kim, C.; Wise, J. A. Influence of $\beta$-Alanine Supplementation on Skeletal Muscle Carnosine Concentrations and High Intensity Cycling Capacity. Amino Acids 2007, 32, 225-233.

39. Lively, C. R.; Feinberg, B. A.; McFarland, J. T. Electrostatic Effect upon Association of Reduced Nicotinamide Adenine Dinucleotide and Equine Liver Alcohol Dehydrogenase. Biochemistry 1987, 26, 5719-5725. 40. Sun, J. J.; Xu, J. J.; Fang, H. Q.; Chen, H. Y. Electrocatalytical Oxidation of NADH with Dopamine Covalently Bound to Self-Assembled Cysteamine Monolayers on a Gold Electrode. Bioelectrochem. Bioenerg. 1997, 44, 45-50.

41. Wightman, R. M.; May, L. J.; Michael, A. C. Detection of Dopamine Dynamics in the Brain. Anal. Chem. 1988, 60, 769A-793A.

42. Park, D. H.; Kim, S. K.; Shin, I. H.; Jeong, Y. J. Electricity Production in Biofuel Cell Using Modified Graphite Electrode with Neutral Red. Biotechnol. Lett. 2000, $22,1301-1304$. 


\section{Table of Contents artwork}

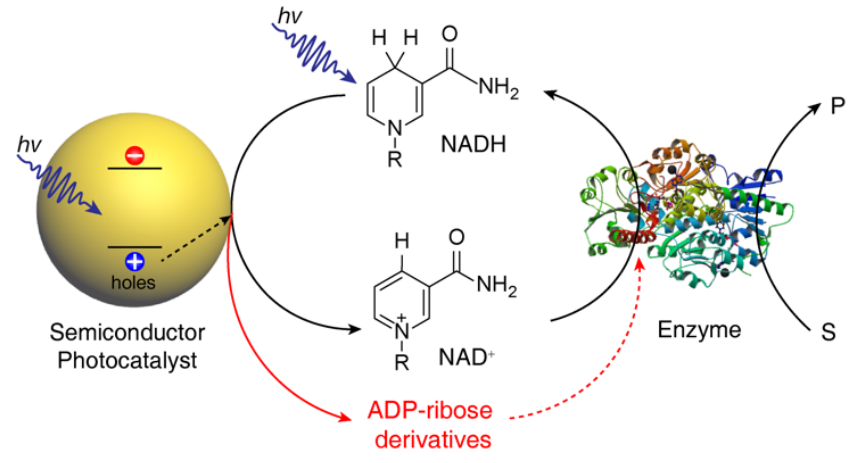

